\title{
28 Research Square \\ The Impact of the COVID-19 Outbreak on Intensive Care in Northern Saitama Japan
}

\section{Ken Nakamura ( $\nabla$ cardiacsurgken@ybb.ne.jp )}

Saitama Cardiovascular and Respiratory Center

Kouan Orii

Saitama Cardiovascular and Respiratory Center

\section{Taichi Kondo}

Saitama Cardiovascular and Respiratory Center

\section{Mitsutaka Nakao}

Saitama Cardiovascular and Respiratory Center

\section{Makoto Wakatabe}

Saitama Cardiovascular and Respiratory Center

\section{Research Article}

Keywords: COVID-19, Intensive care, incident report, emergency patients, overtime work

Posted Date: September 1st, 2021

DOI: https://doi.org/10.21203/rs.3.rs-778267/v1

License: (c) (1) This work is licensed under a Creative Commons Attribution 4.0 International License. Read Full License 


\section{Abstract \\ Background}

We examined and compared the actual impact of COVID-19 on local medical care in northern Saitama Japan, especially in intensive care units (ICUs), before (2019) and during (2020) the pandemic.

\section{Methods}

The impact of COVID-19 on emergency care responses was compared with acceptances and refusals in 2019 and 2020. We also examined the number of surgeries performed by ICU surgical departments. The impact on intensive care was examined regarding the numbers of incident reports and the severity percentage calculated from the integrated team medical care and safety system. We also compared the overtime work of physicians working.

\section{Results}

In 2019, there were 2,136 emergency patient requests, and 1,811 patients were received. In contrast, in 2020 , there were 2,371 emergency patient requests, and 1,822 patients were accepted, representing a decrease of $76 \%(p=0 \rrbracket 931)$. There were significantly more refusals in 2020, $303(14 \rrbracket 1 \%)$ in 2019 and $506(21 \otimes 3 \%)$ in $2020(p=0 \otimes 0004)$. In 2020 , the number of surgeries increased in neurosurgery, cardiac surgeries, and vascular surgeries and over time increased in all surgical units. There were 396 incidents reported in ICUs in 2019; this increased significantly to 510 in 2020 ( $p=0 ख 001)$.

\section{Conclusion}

Even though intensive care management was restricted, the number of patients and doctors' overtime work increased compared to before the spread of COVID-19, and the surrounding environment led to an increase in the number of incidents. The environments in ICUs must be actively improved to prepare for an even more severe situation in the future.

\section{Introduction}

In this unprecedented pandemic era, the entire human race has become frightened by the threat of COVID19 , and healthcare workers are constantly forced to exposed to patients infected with COVID-19 in their daily work. In contrast, patients other than those infected by COVID-19 who require urgent treatment have not been receiving sufficient medical care in the midst of the spread of the new virus.

Throughout Japan, the term "healthcare shortage" has been used to describe the situation, but it is questionable to what extent the current situation has been recognized. Our hospital, located in the 
northern part of Saitama Prefecture in Japan, provides emergency medical services to a population of approximately 600,000 people, including those in the surrounding areas; however, there are currently no statistical data on the present impact of the virus in such a local area.

We investigated the impact of COVID-19 on the intensive care and emergency care of patients in surgical treatment units (mainly cardiac surgery, vascular surgery, neurosurgery, and respiratory surgery) in 2020. We did so because the acceptance of emergency patients was no longer a matter that was decided on the basis of the department's spare capacity as it was before the COVID-19 pandemic. Because in our hospital, we have dedicated personnel and a department for COVID-19, and our emergency medical system gives top priority to accepting patients with COVID-19. The availability of services for other emergency patients depends on the patients with COVID-19.

The respiratory disease intensive care unit (RCU) plays a role in handling patients with severe coronary disease, while other surgical intensive care patients are mainly treated in the intensive care unit (ICU). The RCU nurses are chiefly responsible for the care of patients with COVID-19, but the ICU nurses support them during staffing difficulties.

The bed occupancy status in the RCU for patients with COVID-19 may not have been full at all times due to the impact of the emergency declaration throughout the year. The number of nurses needed changed according to the COVID-19 transmission at that time and the status of support nurses from the ICU also fluctuated.

Therefore, the number of ICU beds depends on the number of nurses supporting the RCU and currently fluctuates from a maximum of 10 beds to a minimum of four beds. Since the number of beds available for intensive care fluctuates in a COVID-19 environment, the impact of bed control on the prognosis of patients is significant, and the emergency system for patients without COVID-19 is inevitably limited, contributing to a tightening of regional medical care.

\section{Material And Methods}

In this study, we examined the impact of COVID-19 (2020) on the intensive care system compared to before the pandemic (2019). In the field of emergency medicine, we compared the impact of COVID-19 on emergency response in terms of the number of requests for emergency patient admissions, the actual number of acceptances, and the refusal rate in 2019

(January-December) and 2020 (January-December). We also examined the number of surgeries performed by the surgical departments in the ICU during this period. In addition, we compared the overtime work of physicians who engaged in intensive care from each department during the same period.

The impact on intensive care, including in the ICU, under the support system of nurses was examined in terms of the total number of incident reports and severity percentage calculated from the integrated team 
medical care and safety system (Safe Master (TM) (Safe Master Inc., Fukuoka). The incident levels followed the categories by Safe Master ${ }^{\circledR}$ and are shown in Table 1.

Data Collection

The data were managed by the Saitama Prefecture Emergency Medical Information System. Written informed consent was obtained from all patients.

\section{Statistical Analysis}

All data were analyzed using IBM SPSS Statistics for Windows (version 26.0; IBM Corp., Armonk, NY, USA). Continuous variables were presented as mean \pm standard deviation. The normality of all variables was assessed using the Shapiro-Wilk test. A paired t-test was used for comparison between the two counties in 2019 (January to December) and 2020 (January to December). Statistical significance was defined as a probability value of $p<\varangle 05$.

\section{Results}

The total number of emergency patients, actual acceptances, and refusals in 2019 and 2020 are shown in Table 2. In 2019, the total number of emergency patient requests was 2,136, and the total number of patient acceptances was 1,811 (85\%). In contrast, in 2020 , the total number of emergency patient

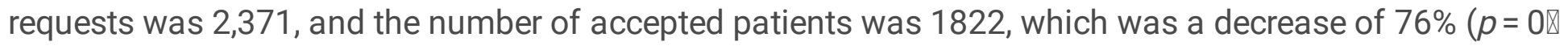
931). As a result of including the floating number of emergency call cancellations, call failures, and requests for instructions in the actual acceptances number of emergency patients, the number of refused acceptances was significantly higher in 2020, from $303(14 \rrbracket 1 \%)$ in 2019 to $506(21 \rrbracket 3 \%)(p=0 \rrbracket 0004)$.

In 2020, the number of surgeries increased in neurosurgery, cardiac surgery, and vascular surgery (Fig. 1). Overtime hours increased in neurosurgery, cardiac surgery, vascular surgery, cardiology, respiratory surgery, and gastrointestinal surgery (Fig. 2). The number of incident reports registered in Safe Master

and the severity of the incidents are shown in Table 3 and Fig. 3 . The number of incidents reported in the ICU in 2019 was 396, while it increased significantly to 510 in $2020(p=0 ख 001)$.

The incidence level 0 was significantly higher in 2020 when comparing the monthly means $(p=0 \otimes 006)$. As shown in Table 3 and Fig. 3, reports of and over the level of 3b, which require intense treatment, increased to seven in 2020 , but this was not significant.

\section{Discussion}

In 2020, hospitals all over Japan started giving top priority to patients with COVID-19 in their emergency systems, while emergency responses for other patients became a problem in every region. Our hospital, located in the northern part of Saitama Prefecture, was no exception, and prioritized patients with COVID19 while also responding to emergency calls from general emergency patients whenever possible. Our 
hospital is one of the few in the country that actively accepts patients with COVID-19 as well as other patients in severe conditions. As the northern part of Saitama Prefecture is a depopulated area with few hospitals, the need for local medical care is higher than in urban areas. It has been reported that the number of emergency admissions for non-COVID-19 infections decreased among all admissions to Veterans Affairs hospitals ${ }^{1}$ along with the number of cardiac catheterizations. ${ }^{2}$ However, this phenomenon does not apply to our hospital, as it is in a depopulated area.

Even in 2020, when COVID-19 infections were widespread, the total number of emergency patient requests increased, which confirmed the development of community-based emergency medical care. At the same time, being a regional COVID-19 institution hospital, the rate of emergency refusal increased compared to 2019. A similar situation led to an increase in out-of-hospital cardiac arrests in Italy. ${ }^{3}$ Moreover, for unknown reasons, the number of operations in surgical departments, the main users of the ICUs, increased in 2020, and these patients required intensive care management. In the future, it will be necessary to ensure the prompt availability of services for emergency patients without COVID-19.

Overtime tended to be more common for doctors working in neurosurgery, cardiac surgery, and vascular surgery, where the ICUs are predominantly used. A study of the impact of such a situation on the ICU showed some surprising results compared to the situation before the spread of the COVID-19 infections in 2019. ${ }^{4}$ The number of incidents reported in our intensive care units (ICUs, RCUs) increased significantly in 2020 compared to 2019. One of the reasons for this was the inadequate management system for general critically ill patients. Intensive care for patients with COVID-19 requires special care, and medical personnel must take all possible precautions; hence, use of the ICUs for critically ill patients other than patients with COVID-19 had to be restricted due to a lack of manpower. However, the level of events was low in the majority of the cases, indicating that a minimum level of control could be maintained even in the context of a high acceptance rate of patients with COVID-19 infections.

Contrary to this result, the number of surgeries on general critical care patients increased even during the COVID-19 pandemic, reflecting the high need for critical care management and overtime hours worked by doctors. It is possible that the increase in the number of overtime hours worked by doctors was influenced by the overload of the outbreak, which was not part of the normal system of care.

Our results show that the environment in our region is quite difficult and that doctors, nurses, and other medical staff work at the limit of their abilities. However, efforts to treat other emergencies, including COVID-19 infections, cannot be stopped, and comprehensive national measures are needed to prevent the collapse of community healthcare.

The Japanese Ministry of Health, Labour and Welfare aims to reform of the way doctors work and has established a special level of overtime to manage the problem of the uneven distribution of doctors in rural areas after April 2024. However, this problem will not be solved unless the COVID-19 pandemic improves. ${ }^{5}$ In addition to working hours, it is also important to ensure special infection prevention measures for healthcare workers associated with COVID-19, especially those involved in intensive care. ${ }^{6}$ 
Phua et al. insisted that hospital administrators, governments, and policy makers must work with ICU practitioners to prepare for the substantial increase in critical care bed capacity and must protect healthcare workers from nosocomial transmission, physical exhaustion, and mental health issues. ${ }^{7}$

A challenge for the future is to improve the medical system and reduce the total number of incidents, even in situations where COVID-19 infections are widespread.

\section{Limitation}

The northern part of Saitama Prefecture is also a depopulated medical area, but this study is limited in its generalizability of the entire area because the data was collected at a single-institution analysis. In addition, since we are comparing the effects of COVID-19 over a 2-year period, a longer observation period is needed.

\section{Conclusion}

We investigated the current situation of intensive care during the COVID-19 pandemic in northern Saitama Prefecture. Even though intensive care management was restricted, the number of patients and doctors' overtime work increased due to the pandemic, and the surrounding environment led to an increase in the number of incidents. It is necessary to actively improve the environment of ICUs to prepare for an even more severe situation in the future.

\section{Declarations}

Author contributions: K. N. and K. O. made a substantial contribution to the concept and design of the work and acquisition, analysis,and interpretation of data. K. N., T. K., and K. O. drafted the article and revised it critically for important intellectual content. T. K. and M. N. provided administrative support. K. N. and K. O. provided the study materials and access to the patients. K. N. collected and assembled the data. K. N. and T. K. analyzed the data and interpreted the results. All authors helped in the manuscript writing and approved of the version to be published.

Conflict of interest: The authors have no conflicts of interest to declare.

Funding: None

Ethical statement: The authors are accountable for all aspects of the work in ensuring that questions related to the accuracy or integrity of any part of the work were appropriately investigated and resolved. This study conformed to the provisions of the Declaration of Helsinki and all its amendments. This study was approved by the Institutional Review Board of the Saitama Cardiovascular and Respiratory Center (No. 2021013). Written informed consent was obtained from the patients for use of the data for academic publication purposes if the patient needed an operation. 
Consent for publication: Not applicable

Availability of data and materials: [Not applicable]

Acknowledgments: [Not applicable]

Reporting checklist statement: We present the following article/case in accordance with the STROBE reporting checklist.

\section{References}

1. Baum A, Schwartz MD. Admissions to veterans affairs hospitals for emergency conditions during the COVID-19 pandemic. JAMA 2020; 324(1): 96-9. doi:10.1001/jama.2020.9972

2. Kadavath S, Mohan J, Ashraf S, et al. Cardiac catheterization laboratory volume changes during COVID-19 - findings from a cardiovascular fellows consortium. Am J Cardio/2020; 130: 168-9. doi:1016/j.amjcard.2020.06.009

3. Baldi E, Sechi GM, Mare C, et al. Out-of-hospital cardiac arrest during the COVID-19 outbreak in Italy. N Engl J Med 2020; 383: 496- doi:10.1056/NEJMc2010418

4. Murthy S, Gomersall CD, Fowler RA. Care for critically ill patients with COVID-19. JAMA 2020; 323(15): 1499- doi:10.1001/jama.2020.3633

5. Materials from the 159th Working Conditions Subcommittee of the Labour Policy Council (held on 10 January 2020) and materials from the 7th Study Group on the Promotion of Workplace Reform for Doctors. https://www.mhlw.go.jp/stf/newpage_10091.html

6. Arabi Y, Murthy S, Webb S. COVID-19: a novel coronavirus and a novel challenge for critical care. [published correction appears in Intensive Care Med. 2020 Mar 18]. Intensive Care Med 2020; 46(5): 833-6. doi: 1007/s00134-020-05955-1

7. Phua J, Weng L, Ling L, et al. Intensive care management of coronavirus disease 2019 (COVID-19): challenges and recommendations. Lancet Respir Med 2020 May; 8(5): 506- doi:10.1016/S22132600(20)30161-2.Epub 2020 Apr 6.

\section{Tables}


Table 1. Incident levels follow the categories of the integrated team medical care and safety system.

\section{Incident Level}

$0: 0.01$ Even if it had been implemented, the impact on patients would have been small (no treatment required).

0.02 If implemented, the impact on patients would be considered moderate (requiring treatment).

0.03 If implemented, the effects on the body would be significant (potentially life-threatening).

1 : Conducted, but no real harm to patients (some impact cannot be ruled out)

2 : No treatment or therapy was given (the need for increased patient observation, minor changes in vital signs, and safety checks arose).

3a : Required simple procedures and treatments (disinfecting, compressing, suturing skin, administering painkillers, etc.)

3b : Required intensive treatment or therapy (e.g., severe change in vital signs, placement on a ventilator, surgery, extended hospital stay, outpatient admission, fracture)

4a : Permanent disability or sequelae, but not accompanied by significant functional impairment or cosmetic problems

$4 \mathrm{~b}$ : Permanent disability and sequelae, with no significant functional impairment or cosmetic problems

5 : Death (excluding death due to the natural course of the primary disease)

$\mathrm{X} 1$ : Impact on the patient cannot be determined

Table 2. Total number of emergency patients, actual acceptances, and refusals for 2019 and 2020.

\section{Emergency Patient Response（2019 vs 2020 )}

$\begin{array}{cccccc}\begin{array}{c}\mathrm{N} \\ \text { (Number of people) }\end{array} & \begin{array}{c}2019 \\ \text { (Monthly average) }\end{array} & \begin{array}{c}2019 \\ \text { (Yearlytotal) }\end{array} & \begin{array}{c}2020 \\ \text { (Monthly average) }\end{array} & \begin{array}{c}2020 \\ \text { (Yearly total) }\end{array} & \text { P } \\ \text { Emergency requests } & 178 \pm 27 & 2136 & 197 \pm 22 & 2371 & 0.078 \\ \text { Actual acceptances } & 150 \pm 28 & 1811(85 \%) & 151 \pm 16 & 1822(76 \%) & 0.931 \\ \text { Refusal } & 25 \pm 8.5 & 303(14.1 \%) & 42 \pm 12 & 506(21.3 \%) & 0.0004\end{array}$

Table 3. Number of monthly incident reports registered in Safe Master and the severity of all incidents. 


\section{The number of monthly incident reports (2019 VS 2020)}

$\begin{array}{cccccc}\text { Incident Level } & 2019 \text { (a month) } & \text { Yearly total } & 2020 \text { (a month) } & \text { Yearly total } & \text { P } \\ 0 & 4.3 \pm 3.3 & 52 & 10.3 \pm 5.9 & 124 & 0.006 \\ 1 & 16.7 \pm 4.2 & 201 & 18.8 \pm 4.1 & 226 & 0.20 \\ 2 & 8.1 \pm 2.3 & 98 & 8.8 \pm 3.1 & 106 & 0.56 \\ 3 \mathrm{a} & 3.5 \pm 2.6 & 42 & 3.9 \pm 2.1 & 47 & 0.60 \\ \text { 3b } & 0.25 \pm 0.45 & 3 & 0.58 \pm 0.79 & 7 & 0.16 \\ 4 \mathrm{a} & 0 & 0 & 0 & 0 & \\ 4 \mathrm{~b} & 0 & 0 & 0 & 0 & \\ 5 & 0 & 0 & 0 & 0 & \\ \text { Total } & 33 \pm 6.1 & 396 & 42 \pm 8.7 & 510 & 0.001\end{array}$

Figures

Comparison of the Number of Operations（2019 VS 2020)

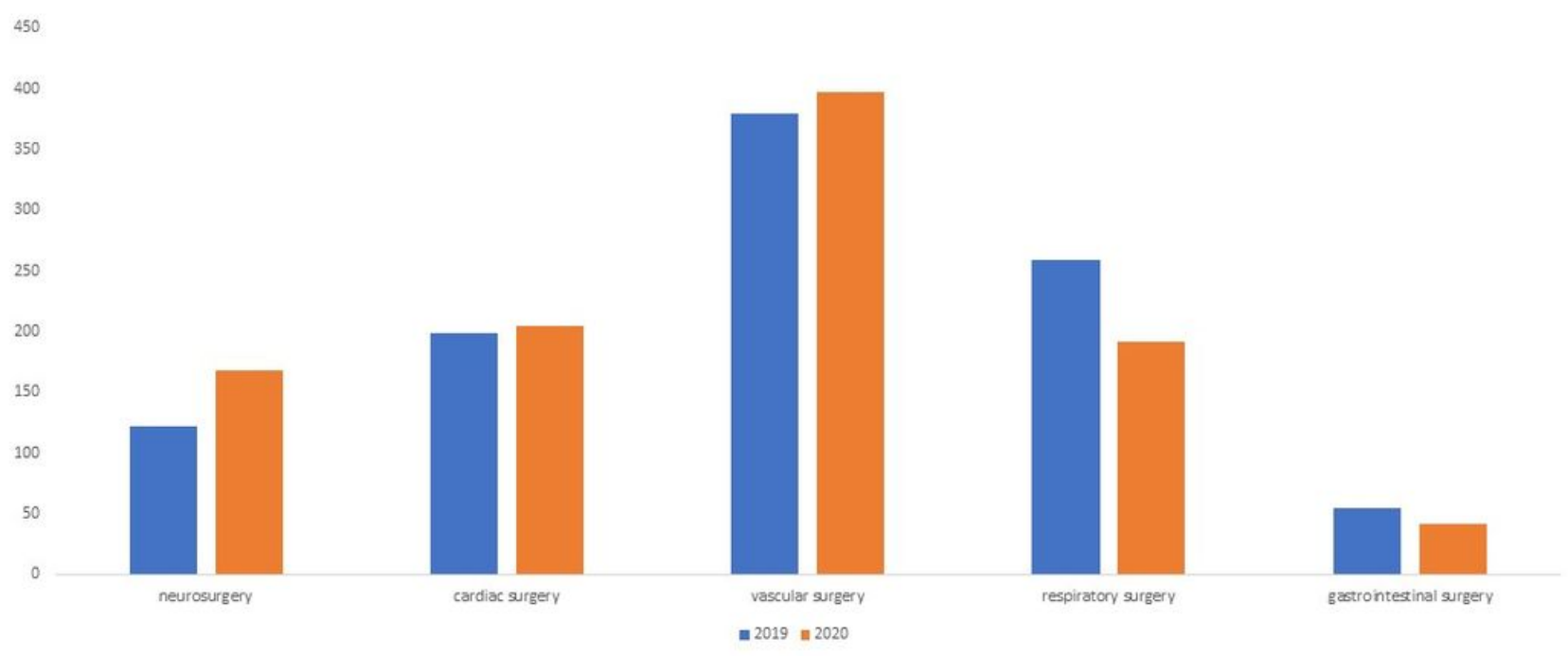

Figure 1.

Figure 1 
Comparison of the number of operations.

\section{Comparison of the Number of Overtime Hours (2019 vs 2020)}

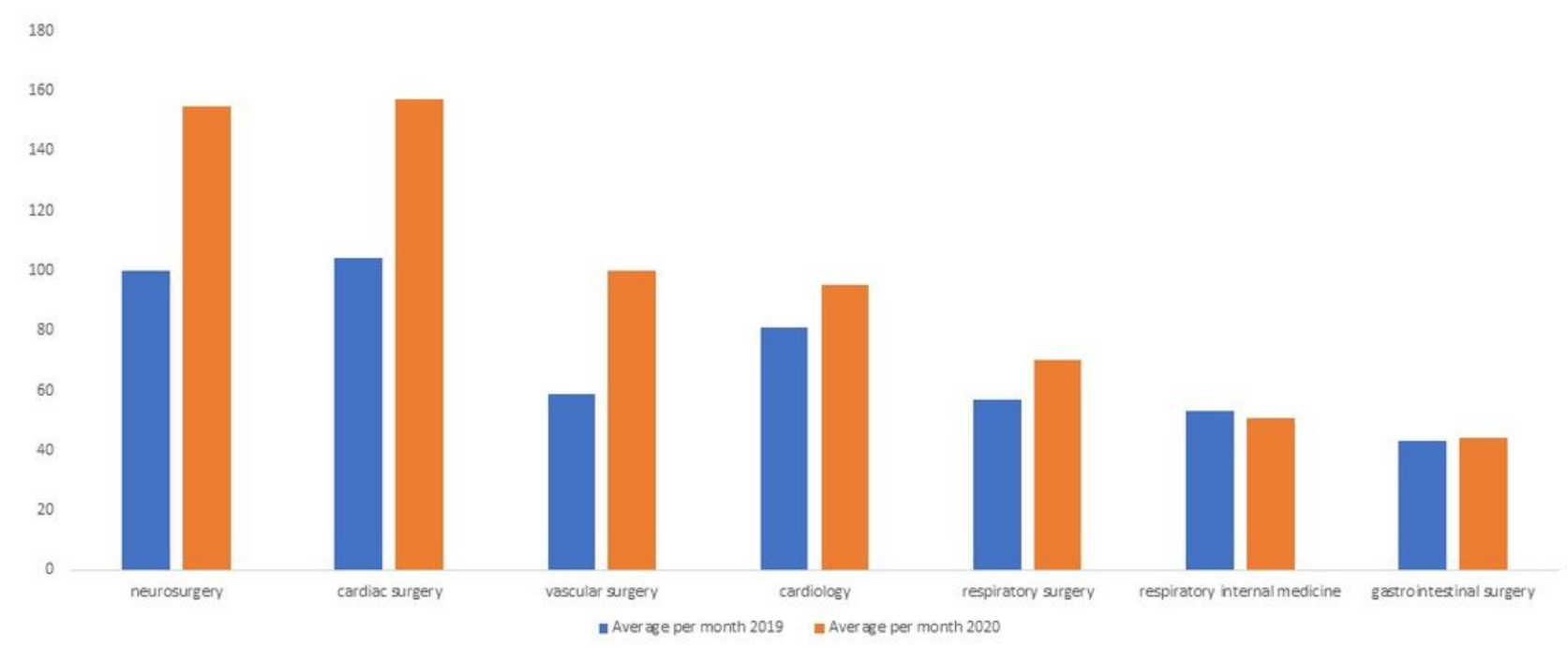

Figure 2.

Figure 2

Comparison of the number of overtime hours.

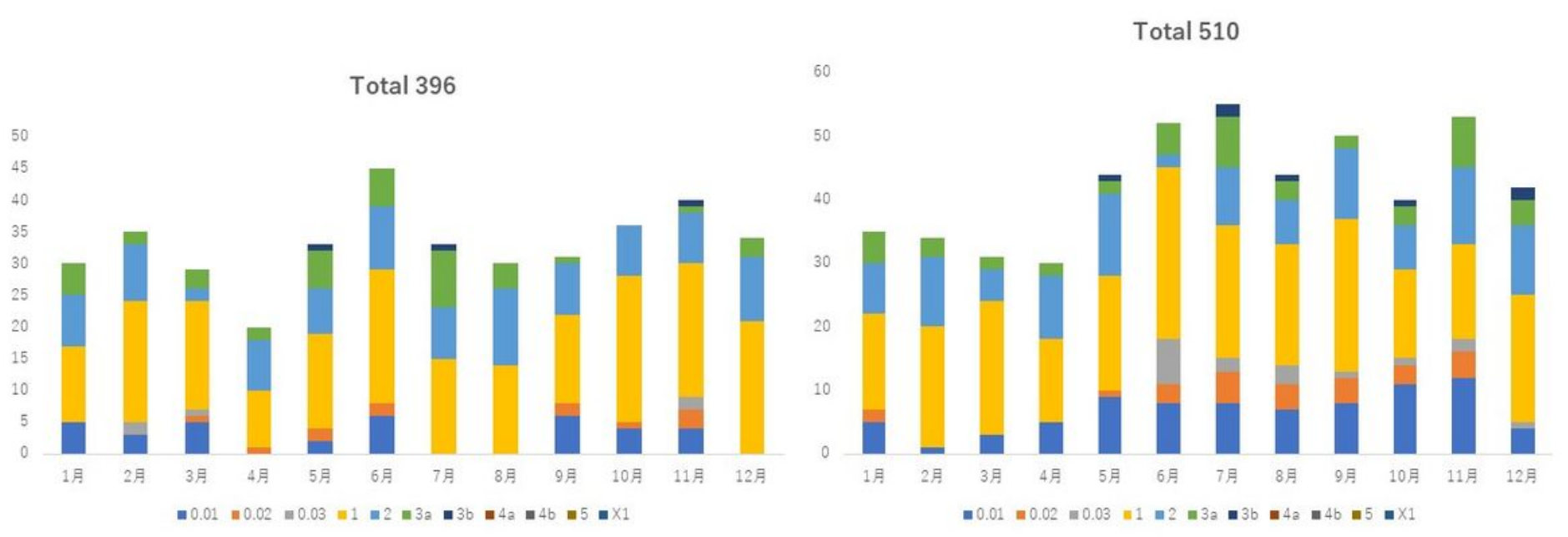

The number of incidents reported in 2019

The number of incidents reported in 2020

Figure 3. 
Figure 3

Incident reports in 2019 and 2020. 